\title{
Renormalization in the Complete Mellin Representation of Feynman Amplitudes
}

\author{
C. de Calan, F. David ${ }^{\star}$, and V. Rivasseau \\ Centre de Physique Théorique de l'Ecole Polytechnique, F-91128 Palaiseau Cedex, France
}

\begin{abstract}
The Feynman amplitudes are renormalized in the formalism of the CM representation. This Mellin-Barnes type integral representation, previously introduced for the study of asymptotic behaviours, is shown to have the following interesting property: in contrast with the usual subtraction procedures, the renormalization leaves the $\mathrm{CM}$ integrand unchanged, and only results into translations of the integration path. The explicit CM representation of the renormalized amplitudes is given. In addition, the dimensional regularization and the extension to spinor amplitudes are sketched.
\end{abstract}

\section{Introduction}

The complete Mellin (CM) representation of Feynman amplitudes was introduced in [1]. It generalized the multiple Mellin technique [2] in a systematic way which presents great advantages: in [2] indeed, one had first to divide the integration domain of the Schwinger representation into the so-called Hepp sectors [3], so that the desingularization of the integrals was performed in a mixed way, partly with the Hepp variables and partly with the Mellin representation. On the contrary, the CM representation avoids the Hepp sectors splitting and gives the same results in a much simpler way. Let us summarize the main features and the most interesting properties of this representation.

For any Feynman graph, the related Feynman amplitude is written as an integral of the Mellin-Barnes type : the CM integrand is a product of $\Gamma$-functions with linear arguments, and of linear powers of the external invariants and internal masses of the Feynman graph, while the integration path is the set of the imaginary axes. The linear polar varieties of the integrand form a lattice in the real hyperplane, depending only on the topology of the graph.

Thus the Cauchy theorem gives an easy determination of any asymptotic expansion, by translations of the integration path. This explicit study of the asymptotic behaviours, which motivated at first the CM representation, is explained in [1], some references and physical examples being quoted in [2].

* Service de Physique Théorique, CEN Saclay, F-91190 Gif-sur-Yvette, France 
Another interesting property is that the renormalization takes a very simple form in the CM representation: it only results in translations of the integration path, without any change in the integrand. This was conjectured in [1], on the basis of simple examples. The main result of the present paper is to prove it, and to give explicitly the CM representation of the renormalized Feynman amplitudes. The fact that the integrand is unmodified allows to use the same method as in the convergent case, for the determination of asymptotic expansions. More generally, this fact is a nice property for all studies concerned with the dependence on the invariants (algebraic properties, differential equations etc...).

On the other hand, we think that the CM representation could be useful to the study of other topics: analytic renormalization [4], dimensional renormalization, Landau singularities. With regard to the dimensional regularization, we give some indications in our last section. The problem of the Landau singularities is not studied here: we use the euclidean metric throughout; the minkowskian case may then be reached by a Wick rotation, raising the question of the convergence at infinity of the $\mathrm{CM}$ integrals.

Finally we give the plan of our paper. In Sect. II, we recall the definition of the CM representation in the convergent case, and the notations. In Sect. III, we perform the renormalization and state the explicit $\mathrm{CM}$ representation of the renormalized Feynman amplitudes. Section IV gives further topics, namely the analytic continuation in dimension, and the extension of our representation to the case of particles with spin, and to the case of derivative couplings (for simplicity, we work in Sect. II and III with scalar amplitudes).

\section{CM Representation of the Convergent Feynman Amplitudes}

In this section, we mainly recall the results of [1] and the notation used in the following sections. Given any Feynman graph $G$ with $L_{G}$ independent loops, we label its $l_{G}$ internal lines by $i$, the internal masses by $m_{i}$, its distinct one-trees (connected trees spanning all vertices of $G$ ) by $j$, its distinct two-trees (trees with two connected components, spanning all vertices of $G$ ) by $k$, the cut-invariant corresponding to any two-tree $k$ by $s_{k}$ (for different two-trees, the invariants $s_{k}$ may coincide). Introducing $u_{i j}$ (respectively $u_{i k}$ ) $=0$ or 1 following the line $i$ belongs or not to the one-tree $j$ (respectively two-tree $k$ ), the Symanzik polynomials are:

$$
\begin{aligned}
& U=\sum_{j} U_{j}=\sum_{j} \prod_{i} \alpha_{i}^{u_{i j}} \\
& N=\sum_{k} N_{k}=\sum_{k} s_{k} \prod_{i} \alpha_{i}^{u_{i k}}
\end{aligned}
$$

and the Feynman amplitude is given by the $\alpha$ representation:

$$
F\left(s_{k}, m_{i}^{2}\right)=\int_{0}^{\infty} \prod_{i} d \alpha_{i} e^{-\sum_{i} \alpha_{i} m^{2} i} U^{-D / 2} e^{-N / U},
$$

where $D$ is the space-time dimension. The Feynman integrand may then be expressed by a Mellin representation:

$$
U^{-D / 2} e^{-N / U}=I_{C_{0}}=\int_{C_{0}} \frac{\prod_{j} \Gamma\left(-x_{j}\right)}{\Gamma\left(-\sum_{j} x_{j}\right)} \prod_{k} s_{k}^{y_{k}} \Gamma\left(-y_{k}\right) \prod_{i} \alpha_{i}^{\varphi_{i}-1},
$$


where the complex variables $x, y$ belong to the cell $C_{0}$ :

$$
C_{0}=\left\{x, y \mid \sum_{j} x_{j}+\sum_{k} y_{k}=-\frac{D}{2} ; \operatorname{Re} x_{j}<0 \forall j ; \operatorname{Re} y_{k}<0 \forall k\right\} .
$$

The integration symbol means

$$
\int_{-\infty}^{+\infty} \prod_{j} \frac{d \operatorname{Im} x_{j}}{2 i \pi} \prod_{k} \frac{d \operatorname{Im} y_{k}}{2 i \pi} 2 i \pi \delta\left(\sum_{j} \operatorname{Im} x_{j}+\sum_{k} \operatorname{Im} y_{k}\right)
$$

and

$$
\varphi_{i}=\sum_{j} u_{i j} x_{j}+\sum_{k} u_{i k} y_{k}+1 .
$$

The convergence of the Feynman integral is equivalent to the superficial convergence of every subgraph $S$ of $G$ :

$$
\omega_{S}=l_{S}-\frac{D}{2} L_{S}>0 \quad \forall S \cong G .
$$

Theorem 1 in [1] says that this convergence is also equivalent to the following assertion :

$$
C_{0} \cap \Delta_{G} \neq \varnothing
$$

where

$$
\Delta_{G}=\left\{x, y \mid \operatorname{Re} \varphi_{i}>0 \quad \forall i \in G\right\} .
$$

This is sufficient to exchange the $x, y$, and $\alpha$ integrations and to obtain the CM representation of the amplitude:

$$
F\left(s_{k}, m_{i}^{2}\right)=\int_{C_{0} \cap \Delta_{G}} \frac{\prod_{j} \Gamma\left(-x_{j}\right)}{\Gamma\left(-\sum_{j} x_{j}\right)} \prod_{k} s_{k}^{y_{k}} \Gamma\left(-y_{k}\right) \prod_{i}\left(m_{i}^{2}\right)^{-\varphi} \Gamma\left(\varphi_{i}\right) .
$$

Conversely let us assume there exists a divergent subgraph $S\left(\omega_{S} \leqq 0\right)$. We define :

$$
\varphi_{S}=\sum_{i \in S} \varphi_{i}=\sum_{j}\left(\sum_{i \in S} u_{i j}\right) x_{j}+\sum_{k}\left(\sum_{i \in S} u_{i k}\right) y_{k}+l_{S} .
$$

For getting any one-tree $j$ or two-tree $k$ of $G$, one must remove at least $L_{S}$ lines from every subgraph $S$ having $L_{S}$ independent loops. Thus:

$$
\sum_{i \in S} u_{i j}=L_{S}+a_{S j} ; \quad \sum_{i \in S} u_{i k}=L_{S}+a_{S k},
$$

where $a_{S_{j}}, a_{S_{k}}$ are non-negative integers.

Using $\sum_{j} x_{j}+\sum_{k} y_{k}=-\frac{D}{2}$ we find :

$$
\varphi_{S}=\omega_{S}+\sum_{j} a_{S j} x_{j}+\sum_{k} a_{S k} y_{k}
$$

which shows that $C_{0} \cap \Delta_{G}$ is empty $\left(\omega_{S}, \operatorname{Re} x_{j}, \operatorname{Re} y_{k}\right.$ being not positive). 
In [1] it was shown how the renormalization of the divergence corresponding to a subgraph $S$ may be performed by translations of the integration path. But the generalization to the complete renormalization is not obvious and will be described in the following section.

\section{CM Representation of the Renormalized Divergent Feynman Amplitudes}

We rewrite the Feynman integrand defined in (2) as:

$$
I_{C_{0}}=\int_{C_{0}} \prod_{\nu=1}^{N} \Gamma\left(-z_{v}\right) \prod_{i} \alpha_{i}^{\varphi_{2}-1} f\left(z_{v}\right)
$$

where the $z_{v}$ variables stand for the $x_{j}$ and $y_{k}$ variables,

$$
f\left(z_{v}\right)=\frac{1}{\Gamma\left(-\sum_{j} x_{j}\right)} \prod_{k} s_{k}^{y_{k}}
$$

is analytic, and

$$
C_{0}=\left\{z \mid \sum_{v=1}^{N} z_{v}=-\frac{D}{2} ; \operatorname{Re} z_{v}<0 \forall v\right\} .
$$

We use the renormalization defined by the $\mathscr{R}$ operation of Bergère and Lam [5]:

$$
\mathscr{R} I_{C_{0}}=\left[\prod_{S \subseteq G}\left(1-\mathscr{T}_{S}\right)\right] I_{C_{0}} .
$$

Let us recall how this $\mathscr{R}$ operation applies to any function $g(\alpha)$. For each subgraph $S$, the $\alpha_{i}$ variables, $i \in S$, are scaled by $\varrho_{S}$, and one writes the generalized expansion in $\varrho_{S}$ :

$$
g\left(\alpha ; \varrho_{S}\right)=\sum_{p=p_{\min }}^{+\infty} \varrho_{S}^{p} g_{p}(\alpha) .
$$

Then by definition the $\mathscr{T}_{S}$ operator only retains the beginning of this expansion, namely those terms with $p \leqq-l_{S}$, and one finally takes $\varrho_{S}=1$. If $p_{\min }>-l_{S}$ (superficial convergence of the subgraph $S$ ), one has: $\mathscr{T}_{S} g=0$.

In order to obtain the Taylor expansions of $I_{C_{0}}$, we will use the method described in [1]. By translating the integration path of (11), the various terms of the Taylor expansions are given by the residues of the simple poles of the functions $\Gamma\left(-z_{v}\right)$. And these residues will be written as differences of integrals $I_{C}$ in various regions, or "cells" $C$, in the space of the $z$ variables.

Definition 1. A cell $C$ is defined by a set of non-negative integers $n_{v}(C)$ (one of them at least being zero):

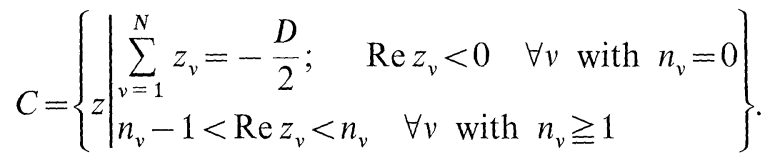

Definition 2. Let $A$ be the set of values taken by the index $v(|A|=N$ is the number of one-trees plus the number of two-trees of the graph). For any strict subset $E$ of $A$ 
$(|E|<N)$, we define a partial ordering of the cells by:

$$
C_{2} \geqq C_{1} \Leftrightarrow \begin{cases}n_{v}\left(C_{2}\right) \geqq n_{v}\left(C_{1}\right) & \forall v \in E \\ n_{v}\left(C_{2}\right)=n_{v}\left(C_{1}\right) & \forall v \notin E .\end{cases}
$$

Definition 3. Given two cells $C_{1}, C_{2}$ with closures $\bar{C}_{1}, \bar{C}_{2}$, we define their relative coefficient $\varepsilon_{C_{1} C_{2}}$ by:

i) if $\exists E, C_{2} \stackrel{E}{\geqq} C_{1} ; \exists F \subset E, C_{2} \stackrel{F}{\unrhd} C_{1}$ and $\bar{C}_{1} \cap \bar{C}_{2} \neq \emptyset$ i.e. if $n_{v}\left(C_{2}\right)=n_{v}\left(C_{1}\right)+\theta_{v E}$ where $\theta_{v E}=\mid \begin{array}{ll}1 & \forall v \in E \\ 0 & \forall v \notin E\end{array}$ then we put

$$
\varepsilon_{C_{1} C_{2}}=(-1)^{|E|}
$$

For the particular case $E=\emptyset$, we have $\varepsilon_{C C}=1 \forall C$.

ii) In every other case, we put $\varepsilon_{C_{1} C_{2}}=0$.

These coefficients satisfy:

$$
\sum_{C \geqq C_{1}} \varepsilon_{C_{1} C}=0 \quad \forall C_{1}, \forall E .
$$

From this identity one can easily deduce the following lemmas:

Lemma 1. Given $E$ and $C_{1} \stackrel{E}{\geqq} C_{0}, C_{2} \stackrel{E}{\geqq} C_{0}$, where $C_{0}$ is defined in (12), we have:

$$
\sum_{\substack{C \\ C_{1} \leqq} \sum_{C}^{E^{\prime}} C_{2}} \varepsilon_{C C_{2}}=\delta_{C_{1} C_{2}} \quad \forall E^{\prime} \cong E .
$$

Lemma 2. More generally, given $C_{1} \stackrel{E}{\geqq} C_{0}, C_{2} \stackrel{E}{\geqq} C_{0}$, given a subset $F$ of $E$ and a set $\mathscr{E}$ of cells, such that

$$
C \in \mathscr{E} \Rightarrow C^{\prime} \in \mathscr{E} \quad \forall C^{\prime} \geqq C-F \quad \text { and } \quad \forall C^{\prime} \geqq C-F
$$

then we have:

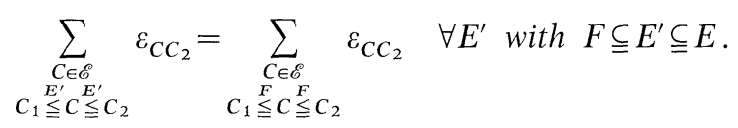

The action of the Taylor operators in the renormalization procedure may now be described in the following way; to each subgraph $S$ corresponds the linear form already given in (10):

$$
\varphi_{S}=\omega_{S}+\sum_{v} a_{S v} z_{v}
$$

where

$$
a_{S v}=\sum_{i \in S} u_{i v}-L_{S} \geqq 0 \forall v .
$$

Moreover $\left\{v \mid a_{S v}=0\right\} \neq \emptyset$ since there are one-trees of $G$, whose restrictions to $S$ are one-trees of $S$. Let us call $E_{S}$ the strict subset of $A$

$$
E_{S}=\left\{v \mid a_{S v}>0\right\}
$$

and $\stackrel{S}{\geqq}$ the corresponding partial ordering $\stackrel{E_{S}}{\geqq}$. 

From Definition 3 , for each cell $C \geqq C_{0}$, with $E_{S} \cong E \subset A, \sum_{C^{\prime} \stackrel{s}{S} C} \varepsilon_{C C^{\prime}}, I_{C^{\prime}}$, is the partial
residue of $I_{C}$ at

$$
z_{\nu}=n_{v}(C) \quad \forall v \in E_{S} .
$$

Thus if the $\alpha_{i}$ 's, $i \in S$, are scaled by $\varrho_{S}$, the Taylor expansion of $I_{C}\left(\varrho_{S}\right)$ is

$$
\left.\sum_{C^{\prime} \leqq} \geqq \sum_{C^{\prime \prime} \stackrel{s}{s} C^{\prime}} \varepsilon_{C^{\prime} C^{\prime \prime}} I_{C^{\prime \prime}}\right) \varrho_{S}^{\varphi_{S}\left(n_{\nu}(C)\right)-l_{S}} .
$$

This expansion may be obtained by writing from Lemma 1 the identity

$$
I_{C}=\sum_{C^{\prime \prime}}\left(\sum_{C \leqq C^{\prime} \leqq C^{\prime \prime}} \varepsilon_{C^{\prime} C^{\prime \prime}}\right) I_{C^{\prime \prime}}
$$

and interchanging the order of summations.

By definition of the Taylor operations, we get:

$$
\mathscr{T}_{S} I_{C}=\sum_{\substack{C^{\prime} \leq ⿱ S \\ \text { sup } \varphi_{S} \leqq 0 \\ C^{\prime}}} \sum_{C^{\prime \prime} \cong C^{\prime}} \varepsilon_{C^{\prime} C^{\prime \prime}} I_{C^{\prime \prime}}
$$

This may be generalized to every nested set of subgraphs:

$$
\mathscr{N}=\left\{S_{1}, \ldots, S_{n}\right\}, \quad S_{1} \subset S_{2} \subset \ldots \subset S_{n} .
$$

Indeed, the Feynman integrand is FINE [2], i.e. it has a simultaneous Taylor expansion in the scaling parameters $\varrho_{S}$ of every nest $\mathscr{N}$. To be explicit, if $\forall S \in \mathscr{N}, \alpha_{i} \rightarrow \varrho_{S} \alpha_{i}, \forall i \in S$, then $U\left(\varrho_{S}\right)=U_{\mathscr{N}} \prod_{S \in \mathscr{N}} \varrho_{S}^{L_{S}}+$ higher order terms, with $U_{\mathscr{N}}=U_{S_{1}} U_{S_{2} / S_{1}} \ldots U_{G / S_{n}}$, the $S_{p} / S_{p-1}$ being the usual reduced graphs.

Let us call $E_{\mathcal{N}}$ the union $\bigcup_{S \in \mathcal{N}} E_{S}$ (and $\geqq$ the corresponding ordering $\left.\stackrel{E_{\mathscr{N}}}{\geqq}\right) . E_{\mathscr{N}}$ is a strict subset of $A$ since for the monomials $j$ of $U_{\mathcal{N}}$

$$
a_{S j}=\sum_{i \in S} u_{i j}-L_{S}=0 \quad \forall S \in \mathscr{N} \Rightarrow j \notin E_{\mathscr{N}}
$$

and the simultaneous Taylor expansion of $I_{C_{0}}$ is

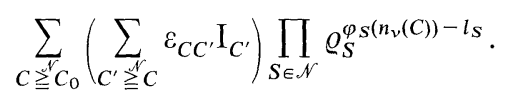

From the definition of the Taylor operations, we retain only those terms for which

$$
\varphi_{S}\left(n_{v}(C)\right)=\sup _{C} \varphi_{S} \leqq 0 \quad \forall S \in \mathscr{N} .
$$

By interchanging the order of summations, we may then state:

\section{Lemma 3.}

$$
\begin{aligned}
\left(\prod_{S \in \mathscr{N}} \mathscr{T}_{S}\right) I_{C_{0}} & =\sum_{C} \mu_{C \mathscr{N}} I_{C}, \\
\mu_{C \mathscr{N}} & =\sum_{C^{\prime} \in \mathscr{E} \mathcal{N}(C)} \varepsilon_{C^{\prime} C}, \\
\mathscr{E}_{\mathscr{N}}(C) & =\left\{C^{\prime} \mid C_{0} \leqq C^{\prime} \leqq C ; \sup _{C^{\prime}} \varphi_{S} \leqq 0 \forall S \in \mathscr{N}\right\} .
\end{aligned}
$$


Remarks. In contrast with the formal infinite series (21) or (24), there is only a finite number of cells $C$ with non-vanishing multiplicity $\mu_{C \mathscr{N}}$ in (25), due to the conditions in

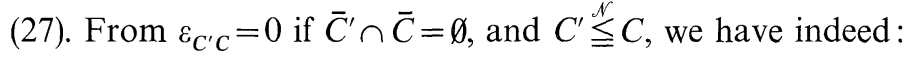

$$
\mu_{C \mathscr{N}}=0 \quad \text { if } \quad \exists S \in \mathscr{N} \quad \text { with } \quad \operatorname{Inf}_{C} \varphi_{S}>0
$$

Moreover, apart from $C_{0}$, the cells with a non-vanishing multiplicity do not lie inside the domain $\left\{\varphi_{S} \leqq 0 \forall S \in \mathscr{N}\right\}$, but only on its border, since from (27) and Lemma 1 we have:

$$
\mu_{C \mathscr{N}}=\delta_{C_{0} C} \quad \text { if } \quad \sup _{C} \varphi_{S} \leqq 0 \quad \forall S \in \mathscr{N} .
$$

We can now give the renormalized integrand, since the $\mathscr{R}$ operation given in (13) can also be written [5]:

$$
\mathscr{R}=\sum_{\mathscr{N}} \prod_{S \in \mathscr{N}}\left(-\mathscr{T}_{S}\right)
$$

where the summation is performed over all the nests, including the empty nest $\mathscr{N}=\emptyset$, for which $\prod_{S \in \emptyset}\left(-\mathscr{T}_{S}\right)=1$. So we state:

\section{Theorem 1.}

$$
\mathscr{R} I_{C_{0}}=\sum_{C} \mu_{C} I_{C}
$$

where

$$
\mu_{C}=\sum_{\mathscr{N}}(-1)^{|\mathcal{N}|} \mu_{C \mathscr{N}}
$$

and $\mu_{C \mathscr{N}}$ is defined in Lemma 3.

Before proceeding to the second step, that is the $\alpha$ integrations, we must summarize some of the results of Bergère and Lam [5].

Let $\mathscr{M}$ be a fixed maximal nest of subgraphs $R_{m}$ of $G$ :

$$
\mathscr{M}=\left\{R_{1}, R_{2}, \ldots, R_{l_{G}}\right\}, \quad R_{m+1}=R_{m} \cup\left\{i_{m+1}\right\} .
$$

For a given $\mathscr{M}$, the set of all nests $\mathscr{N}$ can be split into equivalence classes. Each class $\Gamma$ is characterized by its maximal nest $\mathscr{M}(\Gamma)$ which is the union of two disjoint subnests $\mathscr{H}(\Gamma)$ and $\mathscr{K}(\Gamma)$ such that:

i) $\mathscr{N} \in \Gamma \Leftrightarrow \mathscr{K}(\Gamma) \subseteq \mathscr{N} \cong \mathscr{K}(\Gamma) \cup \mathscr{H}(\Gamma)=\mathscr{M}(\Gamma)$.

Therefore:

$$
\sum_{\mathscr{N} \in \Gamma} \prod_{S \in \mathcal{H}}\left(-\mathscr{T}_{S}\right)=\prod_{K \in \mathscr{K}(\Gamma)}\left(-\mathscr{T}_{K}\right) \prod_{H \in \mathscr{H}(\Gamma)}\left(1-\mathscr{T}_{H}\right)
$$

ii) To each $R_{m} \in \mathscr{M}$ and each class $\Gamma$ correspond subsets $\left\{H_{m j}\right\}$ of $\mathscr{H}(\Gamma),\left\{K_{m j}\right\}$ of $\mathscr{K}(\Gamma)$ satisfying

$$
\begin{aligned}
K_{m j} & \subset H_{m j} \quad \forall j \\
\left(H_{m j}-K_{m j}\right) & \cap\left(H_{m j^{\prime}}-K_{m j^{\prime}}\right)=\emptyset \quad \forall j \neq j^{\prime} \\
R_{m} & =\bigcup_{j}\left(H_{m j}-K_{m j}\right)
\end{aligned}
$$


which means with our notations:

$$
\varphi_{R_{m}}=\sum_{j} \varphi_{H_{m j}}-\sum_{j} \varphi_{K_{m j}} .
$$

Apart from these results, we need the following lemma:

Lemma 4. Given two disjoint nests $\mathscr{A}, \mathscr{B}$, the union of which is a nest $\mathscr{N}$, we have:

$$
\left[\prod_{T \in \mathscr{B}} \mathscr{T}_{T} \prod_{S \in \mathscr{A}}\left(1-\mathscr{T}_{S}\right)\right] I_{C_{0}}=\sum_{C} \mu_{C \mathscr{A} \mathscr{B}} I_{C}
$$

where

$$
\begin{aligned}
& \mu_{C \mathscr{A} \mathscr{B}}=\sum_{C^{\prime} \in \mathscr{E}_{\mathscr{A} \mathscr{B}}(C)} \varepsilon_{C^{\prime} C}, \\
& \mathscr{E}_{\mathscr{A} \mathscr{B}}(C)=\left\{C^{\prime} \mid \begin{array}{c}
C_{0} \leqq C^{\prime} \stackrel{\mathcal{N}}{\leqq} C ; \sup _{C^{\prime}} \varphi_{T} \leqq 0 \forall T \in \mathscr{B} ; \\
\sup _{C^{\prime}} \varphi_{S}>0 \forall S \in \mathscr{A}
\end{array}\right\} .
\end{aligned}
$$

Proof. The proof is by recurrence on the number of subgraphs $S$ in $\mathscr{A}$. For $\mathscr{A}=\emptyset$, Lemma 4 is nothing but Lemma 3 . Let us assume that Lemma 4 is true for $\mathscr{A}^{\prime}=\mathscr{A}-\left\{S_{1}\right\}$ and any second subnest, in particular $\mathscr{B}$ and $\mathscr{B}^{\prime}=\mathscr{B} \cup\left\{S_{1}\right\}$. Then:

$$
\begin{aligned}
& \prod_{T \in \mathscr{B}} \mathscr{T}_{T} \prod_{S \in \mathscr{A}}\left(1-\mathscr{T}_{S}\right) I_{C_{0}} \\
& \quad=\prod_{T \in \mathscr{B}} \mathscr{T}_{T} \prod_{S \in \mathscr{A}^{\prime}}\left(1-\mathscr{T}_{S}\right) I_{C_{0}}-\prod_{T \in \mathscr{B}^{\prime}} \mathscr{T}_{T} \prod_{S \in \mathscr{A}^{\prime}}\left(1-\mathscr{T}_{S}\right) I_{C_{0}} \\
& \quad=\sum_{C} \lambda_{C \mathscr{A} \mathscr{B}} I_{C}
\end{aligned}
$$

with

$$
\lambda_{C \mathscr{A} \mathscr{B}}=\mu_{C \mathscr{A}^{\prime} \mathscr{B}}-\mu_{C \mathscr{A}^{\prime} \mathscr{B}^{\prime}}
$$

But from Lemma 2

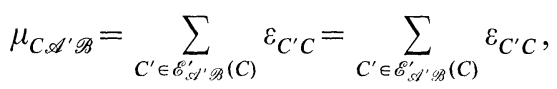

where

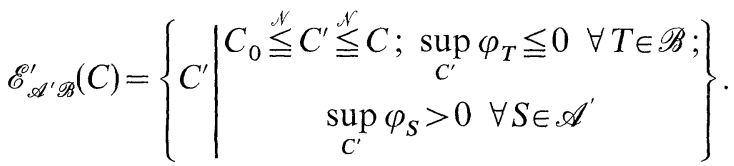

By replacing (40) into (39), one sees $\lambda_{C \mathscr{A} \mathscr{B}}=\mu_{C \mathscr{A} \mathscr{B}}$, which achieves the proof of Lemma 4.

We may now use (30), (31), (33), and Lemma 4 to write:

\section{Lemma 5.}

$$
\mu_{C}=\sum_{\Gamma} \mu_{C \Gamma}
$$


with

$$
\begin{gathered}
\mu_{C \Gamma}=\sum_{\mathscr{N} \in \Gamma}(-1)^{|\mathcal{H}|} \mu_{C \mathscr{N}}=(-1)^{|\mathscr{K}(\Gamma)|} \sum_{C^{\prime} \in \mathscr{E}_{\Gamma}(C)} \varepsilon_{C^{\prime} C}, \\
\mathscr{E}_{\Gamma}(C)=\left\{C^{\prime} \mid \begin{array}{c}
C_{0} \stackrel{\mathscr{M}(\Gamma)}{\leftrightarrows} C^{\prime} \stackrel{M(\Gamma)}{\leqq} C ; \sup _{C^{\prime}} \varphi_{K} \leqq 0 \quad \forall K \in \mathscr{K}(\Gamma) ; \\
\sup _{C^{\prime}} \varphi_{H}>0 \forall \mathrm{H} \in \mathscr{H}(\Gamma)
\end{array}\right\} .
\end{gathered}
$$

Remarks. For each $\Gamma$, there is only a finite number of cells $C$ such that $\mu_{C \Gamma} \neq 0$, since $\mu_{C \Gamma}$ is a finite combination of $\mu_{C \mathscr{N}}$ 's. Moreover many of the $\mu_{C \Gamma}$ 's vanish, since from (43) :

$$
\begin{aligned}
& \mu_{C \Gamma}=0 \quad \text { if } \exists K \in \mathscr{K}(\Gamma) \quad \text { with } \operatorname{Inf}_{C} \varphi_{K}>0 \text {, } \\
& \mu_{C \Gamma}=0 \quad \text { if } \exists H \in \mathscr{H}(\Gamma) \quad \text { with } \operatorname{Sup}_{C} \varphi_{H} \leqq 0 \text {, } \\
& \mu_{C \Gamma}=(-1)^{|\mathscr{H}(\Gamma)|} \delta_{C_{0} C} \quad \text { if } \quad \operatorname{Inf}_{C} \varphi_{H}>0 \forall H \in \mathscr{H}(\Gamma) \text { and } \quad \operatorname{Sup}_{C} \varphi_{K} \leqq 0 \forall K \in \mathscr{K}(\Gamma) .
\end{aligned}
$$

The related vanishing of many $\mu_{C}$ 's also appears in the following lemma:

Lemma 6. For each cell $C$ with $\mu_{C} \neq 0$, we have:

$$
C \cap \Delta_{\mathscr{M}} \neq \emptyset \text {, }
$$

where

$$
\Delta_{\mathscr{M}}=\left\{z \mid \operatorname{Re} \varphi_{\boldsymbol{R}_{m}}>0 \forall R_{m} \in \mathscr{M}\right\}
$$

Proof. If $\mu_{C} \neq 0, \exists \Gamma$ with $\mu_{C \Gamma} \neq 0$. Hence

$$
\exists C^{\prime} \leqq C \quad \text { with } \begin{cases}\varepsilon_{C^{\prime} C} \neq 0 & \\ \sup _{C^{\prime}} \varphi_{H}>0 & \forall H \in \mathscr{H}(\Gamma) \\ \sup _{C^{\prime}} \varphi_{K} \leqq 0 & \forall K \in \mathscr{K}(\Gamma) .\end{cases}
$$

Let $\sigma\left(C^{\prime}\right)$ be the edge of $C^{\prime}: z_{v}=n_{v}\left(C^{\prime}\right) \forall v \in E_{M(\Gamma)}$. From the positivity of the coefficients $a_{S v}$ in $\varphi_{S}, \forall S \in \mathscr{M}(\Gamma)$ :

$$
\begin{aligned}
& \varphi_{H}\left(\sigma\left(C^{\prime}\right)\right)=\sup _{C^{\prime}} \varphi_{H}>0 \\
& \varphi_{K}\left(\sigma\left(C^{\prime}\right)\right)=\sup _{C^{\prime}} \varphi_{K} \leqq 0
\end{aligned}
$$

whence from (34):

$$
\varphi_{R_{m}}\left(\sigma\left(C^{\prime}\right)\right)>0 \quad \forall R_{m} \in \mathscr{M} .
$$

But $C^{\prime} \stackrel{M(\Gamma)}{\leqq} C$ and $\bar{C}^{\prime} \cap \bar{C} \neq \emptyset$ imply $\sigma\left(C^{\prime}\right) \in \bar{C}$.

Thus in the neighbourhood of $\sigma\left(C^{\prime}\right)$ there exist points in $C$ where the $\varphi_{R_{m}}$ are simultaneously positive, which proves Lemma 6. 
Now the result expressed by Lemma 6 is true whatever is the fixed maximal nest $\mathscr{M}=\left\{R_{m}\right\}$ originally choosen. Therefore we shall be able to perform the $\alpha$ integrations with the use of only one further lemma:

Lemma 7. Given a cell $C$, if for each maximal nest $\mathscr{M}, C \cap \Delta_{\mathscr{M}}$ is not empty, then:

$$
\Delta_{C}=C \cap \Delta_{G} \neq \emptyset \text {, }
$$

where

$$
\Delta_{G}=\left\{z \mid \operatorname{Re} \varphi_{i}>0 \quad \forall i \in G\right\} .
$$

Proof. In the space of the Re $z$ 's, any cell $C$ is defined by a set of linear boundary conditions, which we write $\psi_{\beta}>0 \forall \beta$. If in $C$ the $\varphi_{i}$ 's cannot be simultaneously positive, there must exist non-negative real numbers $a_{0}, a_{i}, a_{\beta}$, the $a_{i}$ 's being not all vanishing, with

$$
\sum_{i} a_{i} \varphi_{i}+\sum_{\beta} a_{\beta} \psi_{\beta}+a_{0} \equiv 0
$$

By ordering the $a_{i}$ 's: $a_{i_{m}} \geqq a_{i_{m-1}} \forall m>1$ and putting: $b_{m}=a_{i_{m}}-a_{i_{m-1}}, \quad \forall m>1$; $b_{1}=a_{i_{1}}$ we get a set of non-negative integers $b_{m}$, not all vanishing, with:

$$
\sum_{m} b_{m} \varphi_{R_{m}}+\sum_{\beta} a_{\beta} \psi_{\beta}+a_{0} \equiv 0
$$

where

$$
R_{m}=\left\{i_{m}, i_{m+1}, \ldots, i_{l_{G}}\right\} .
$$

Then there would exist a maximal nest, the $\varphi_{\boldsymbol{R}_{m}}$ of which cannot be simultaneously positive in $C$, which would contradict the hypothesis.

By Lemma 7, the $\alpha$ integrations may now be performed for $z \in \Delta_{C}$ and we set up the results of this section in the following theorem:

Theorem 2. The renormalized Feynman amplitude corresponding to a graph $G$ is given by the $\mathrm{CM}$ representation:

$$
F_{\mathscr{R}}\left(s_{k}, m_{i}^{2}\right)=\sum_{C} \mu_{C} \int_{\Delta_{C}} \frac{\prod_{j} \Gamma\left(-x_{j}\right)}{\Gamma\left(-\sum_{j} x_{j}\right)} \prod_{k} s_{k}^{y_{k}} \Gamma\left(-y_{k}\right) \prod_{i}\left(m_{i}^{2}\right)^{-\varphi_{t}} \Gamma\left(\varphi_{i}\right),
$$

where the multiplicities $\mu_{C}$ are defined in Theorem 1 and Lemma 3, and $\mu_{C}=0$ if $\Delta_{C}=C \cap \Delta_{G}$ is empty.

Remarks. We used in this section the $\mathscr{R}$ operation of Bergère and Lam, and the same machinery of equivalence classes of nests [5]. But it can be noticed that this section gives a proof independent of theirs for the convergence of the renormalized amplitudes. We find $F_{\mathscr{R}}$ as a sum of integrals. However, in contrast with the usual renormalization schemes, the integrands are unmodified, and each of these integrals is separately convergent. The set of cells with non-vanishing multiplicity is a finite set, and these cells lie on the border of $\Delta_{G}$ :

$$
\mu_{C} \neq 0 \Rightarrow\left\{\begin{array}{lll}
\exists z \in C & \text { with } & \operatorname{Re} \varphi_{i}(z)>0 \quad \forall i \\
\exists i & \text { with } & \operatorname{Inf}_{C} \varphi_{i} \leqq 0
\end{array}\right.
$$


Translating the integration path farther inside $\Delta_{G}$ would correspond to performing oversubtractions, which could be expressed in our formalism by the simple replacements :

$$
\begin{aligned}
& \varphi_{S} \rightarrow \varphi_{S}^{\prime}=\varphi_{S}-d_{S} \\
& \Delta_{G} \rightarrow \Delta_{G}^{\prime}=\left\{z \mid \operatorname{Re} \varphi_{S}^{\prime}>0 \quad \forall S \leqq G\right\} .
\end{aligned}
$$

\section{Additional Topics}

\section{Dimensional Regularization}

The CM representation can likely be used to the explicit study of the dimensionally renormalized amplitudes. We do not attempt here to perform the dimensional renormalization, but only give indications on the dimensional regularization. Starting with a convergent Feynman amplitude (for a sufficiently low value of the space-time dimension), let us show how we can reach the following results :

- the amplitude is a meromorphic function of the dimension.

- its dimensional analytic continuation has a CM representation with the same unchanged CM integrand, and again translations of the integration path.

The first of these results is already known (see e.g. [6]), but can be directly proved from the CM representation, in exactly the same way as was determined any asymptotic expansion in [1]. Let $F(D)$ be a Feynman amplitude, convergent for $\operatorname{Re} D<D_{0}$ : it admits a CM representation given by Eq. (8), which we rewrite as :

$$
F(D)=\int_{\gamma_{0} \cap P(D)} \prod_{r} \Gamma\left(\varphi_{r}\right) J(z)
$$

where the $z_{v}$ variables stand for the $x_{j}$ and $y_{k}$ variables as in (11), the linear forms $\varphi_{r}$ stand for $-x_{j},-y_{k}$, and $\varphi_{i}$,

$$
J(z)=\frac{1}{\Gamma\left(-\sum_{j} x_{j}\right)} \prod_{k} s_{k}^{y_{k}} \prod_{i}\left(m_{i}^{2}\right)^{-\varphi_{i}}
$$

is analytic,

$$
\gamma_{0}=\left\{z \mid \operatorname{Re} \varphi_{r}>0 \forall r\right\}
$$

$P(D)$ is the hyperplane $\sum_{v} z_{v}=-\frac{D}{2}$.

The lattice of the polar varieties $\left\{\varphi_{r}=-n_{r}\right\}$, where the $n_{r}$ 's are non-negative integers, gives the boundaries of a set of cells

$$
\gamma=\left\{z \mid \operatorname{Re} \varphi_{r}>0 \text { for } n_{r}=0 ;-n_{r}<\operatorname{Re} \varphi_{r}<-n_{r}+1 \text { for } n_{r} \geqq 1\right\} .
$$

For a given cell $\gamma$, we define $D_{m}$ and $D_{M}$ by:

$$
-\frac{D_{m}(\gamma)}{2}=\sup _{\gamma}\left(\sum_{\nu} \operatorname{Re} z_{\nu}\right) ; \quad-\frac{D_{M}(\gamma)}{2}=\operatorname{Inf}_{\gamma}\left(\sum_{\nu} \operatorname{Re} z_{\nu}\right) .
$$

Then $F_{\gamma}(D)=\int_{\gamma \cap P(D)} \prod_{r} \Gamma\left(\varphi_{r}\right) J(z)$ defines an analytic function of $D$ in the strip $D_{m}<\operatorname{Re} D<D_{M}$. In the same way as in Sect. III of [1], we use the identities 
expressing that $\sum_{v} z_{v}+D_{M}(\gamma) / 2$ belongs to the convex spaces generated by some subsets of the boundary forms of $\gamma$. We obtain:

$$
\left.\frac{1}{\prod_{r}\left(\varphi_{r}+n_{r}\right)}\right|_{P(D)}=\sum_{E} \frac{d_{E}}{\left(D-D_{M}\right)^{q_{E}+1}} \frac{1}{\prod_{r \in E}\left(\varphi_{r}+n_{r}\right)}
$$

where the sets $\left\{\varphi_{r}, r \in E\right\}$ no longer generate $D-D_{M}$, which implies that each term in (61) may be analytically continued to higher values of $D$.

Thus, starting from $\gamma_{0}$, one can explicitly extract the various poles in dimension, and iteratively translate the integration path to other cells. This shows the meromorphy in dimension; the poles in $D$ belong to the discrete set of rational numbers corresponding to the values of $\sum_{v} z_{v}$ at the vertices of the lattice $\left\{\varphi_{r}=-n_{r}\right\}$.

An alternative way of working is the following: we rewrite (59) as a difference of integrals over all the $\operatorname{Im} z_{v}$ 's (no longer linked by the constraint $\left.\sum_{v} z_{v}=-\frac{D}{2}\right)$ :

$$
F(D)=\int_{\gamma_{0} \cap B+(D)} \frac{\prod_{r} \Gamma\left(\varphi_{r}\right)}{\varphi_{0}} J(z)-\int_{\gamma_{0} \cap B-(D)} \frac{\prod_{r} \Gamma\left(\varphi_{r}\right)}{\varphi_{0}} J(z),
$$

where

$$
\begin{aligned}
B_{+}(D) & =\left\{z \mid 0<\operatorname{Re} \varphi_{0}<\varepsilon\right\} \\
B_{-}(D) & =\left\{z \mid-\varepsilon<\operatorname{Re} \varphi_{0}<0\right\} \\
\varphi_{0} & =\sum_{v} z_{v}+\frac{D}{2}
\end{aligned}
$$

and $\varepsilon$ is a small positive real number.

In these integrals, we translate the integration path by increasing the dimension from $D$ to $D^{\prime}$ and crossing the various singularities of the lattice $\left\{\varphi_{r}=-n_{r}\right\}$.

Thus we obtain:

$$
\int_{\gamma_{0} \cap B_{ \pm}(D)} \frac{\prod_{r} \Gamma\left(\varphi_{r}\right)}{\varphi_{0}(D)} J(z)=\sum_{\gamma} \lambda_{\gamma} \int_{\gamma \cap B_{ \pm}\left(D^{\prime}\right)} \frac{\prod_{r} \Gamma\left(\varphi_{r}\right)}{\varphi_{0}\left(D^{\prime}\right)} J(z)+\text { residues. }
$$

In contrast with Sect. III (where we considered only the sub-lattice $\left\{-z_{v}=-n_{v}\right\}$ ), we generally get residues of multiple poles. In any case these residues disappear in the difference (62) if $D^{\prime}$ is such that the domain $\left\{z \mid-\varepsilon<\operatorname{Re} \varphi_{0}\left(D^{\prime}\right)<\varepsilon\right\}$ does not contain any vertex of the lattice. This condition corresponds to fix $\operatorname{Re} D^{\prime}$ between two consecutive poles in dimension. Finally we can come back to the original form of the integral:

$$
F\left(D^{\prime}\right)=\sum_{\gamma} \lambda_{\gamma} \int_{\gamma \cap P\left(D^{\prime}\right)} \prod_{r} \Gamma\left(\varphi_{r}\right) J(z)
$$


The result (64) is similar to that one of our Sect. III : provided that the hyperplane $P\left(D^{\prime}\right)$ does not across the vertices of the lattice $\left\{\varphi_{r}=-n_{r}\right\}$ [that is, for $\operatorname{Re} D^{\prime}$ distinct from the poles of $F(D)]$, then the continued amplitude has a CM representation with unchanged integrand and translated integration path. Of course the multiplicities of the cells in (64) and (56) differ.

To obtain the same result, we could also start from the expression of the regularized amplitude given in [6]. For $\operatorname{Re} D$ between two consecutive poles:

$$
F(D)=\lim _{\mu_{2}^{2}-0} \int_{0}^{\infty} \prod_{i} d \alpha_{i} e^{-\sum_{i} \alpha_{i} \mu_{2}^{2}} \mathscr{R}\left[e^{-\sum_{i} m_{1}^{2} \alpha_{2}} U^{-D / 2} e^{-N / U}\right] .
$$

The bracket in (65) has again a CM representation, with additional variables for the new factor:

$$
e^{-\sum_{i} \alpha_{2} m_{l}^{2}}=\int_{\operatorname{Re} t_{i}<0} \prod_{i}\left[\frac{d \operatorname{Im} t_{i}}{2 i \pi} \Gamma\left(-t_{i}\right)\left(m_{i}^{2}\right)^{t_{i}} \alpha_{i}^{t_{i}}\right] .
$$

Then the $\mathscr{R}$ operation acts in the same way as in Sect. III, with the replacement: $\varphi_{i} \rightarrow \psi_{i}=\varphi_{i}+t_{i}$.

Finally the limit $\mu_{i}^{2} \rightarrow 0$ is easily taken, as explained at the end of Sect. III of [1], giving back (64).

\section{The Case of Non-Scalar Amplitudes}

In this paragraph, we want to generalize our $\mathrm{CM}$ representation to the case of particles with spin, and/or to derivative couplings. In the momentum representation, the Feynman amplitude corresponding to a given graph becomes:

$$
F\left(p_{v}, m_{i}^{2}\right)=\int \prod_{a=1}^{L} d^{D} k_{a} \frac{\mathscr{P}\left(q_{i}\right)}{\prod_{i}\left(q_{i}^{2}+m_{i}^{2}\right)},
$$

where the $p_{v}$ 's are the external momenta, the $k_{a}$ 's are the loop variables, the $q_{i}$ 's are the internal momenta, and $\mathscr{P}$ is a polynomial in the momenta, coming from the derivative couplings and/or from the numerators of the propagators. We take an $\alpha$ representation by:

$$
\frac{q_{i}^{\mu} q_{i}^{v} \ldots q_{i}^{\sigma}}{q_{i}^{2}+m_{i}^{2}}=\int_{0}^{\infty} d \alpha_{i}\left\{\left(-\frac{1}{\alpha_{i}} \frac{\partial}{\partial \zeta_{i}^{\mu}}\right)\left(-\frac{1}{\alpha_{i}} \frac{\partial}{\partial \zeta_{i}^{v}}\right) \ldots\left(-\frac{1}{\alpha_{i}} \frac{\partial}{\partial \zeta_{i}^{\sigma}}\right) e^{-\alpha_{i}\left(q_{i}^{2}+m_{i}^{2}+q_{i} \cdot \zeta_{l}\right)}\right\}_{\zeta_{l}=0}
$$

which gives the following $\alpha$ representation of the amplitude:

$$
F\left(p_{v}, m_{i}^{2}\right)=\int_{0}^{\infty} \prod_{i} d \alpha_{i}\left\{\mathscr{P}\left(-\frac{1}{\alpha_{i}} \frac{\partial}{\partial \zeta_{i}}\right) e^{-\sum_{i} \alpha_{i} \bar{m}_{i}^{2}} U^{-\frac{D}{2}} e^{-\frac{N\left(\bar{s}_{k}\right)}{U}}\right\}_{\zeta_{i}=0} .
$$

Here $\bar{m}_{i}^{2}=m_{i}^{2}-\frac{\zeta_{i}^{2}}{4}$.

$\bar{s}_{k}$ is the cut-invariant of the two-tree $k$, with the external momenta $p_{v}$ replaced by $\bar{p}_{v}=p_{v}+\varepsilon_{v i} \frac{\zeta_{i}}{2}, \varepsilon_{v i}$ being the usual incidence matrix. 
Then we take a CM representation of (69) in the same way as before. For each monomial $\prod_{i}\left(-\frac{1}{\alpha_{i}} \frac{\partial}{\partial \zeta_{i}}\right)^{d_{i}}$ of $\mathscr{P}$ we obtain:

$$
\int_{\Delta}\left\{\prod_{i}\left(-\frac{\partial}{\partial \zeta_{i}}\right)^{d_{\imath}} \frac{\prod_{j} \Gamma\left(-x_{j}\right)}{\Gamma\left(-\sum_{j} x_{j}\right)} \prod_{k} \bar{s}_{k}^{y_{k}} \Gamma\left(-y_{k}\right) \prod_{i}\left(\bar{m}_{i}^{2}\right)^{-\varphi_{t}+d_{t}} \Gamma\left(\varphi_{i}-d_{i}\right)\right\}_{\zeta_{\imath}=0} .
$$

In (70), the action of the differential operators $\frac{\partial}{\partial \zeta_{i}}$ results in translations of the linear forms $-y_{k}$ or $\varphi_{i}-d_{i}$, and factorization of tensors built from the external momenta. The main difference with the scalar case is the complicated list of cells which enter into the new integration domains $\Delta$, depending on the various monomials of $\mathscr{P}$. Otherwise the renormalization will follow the same lines as for the scalar amplitudes.

\section{References}

1. Calan, C., de, Malbouisson, A.P.C.: Ann. Inst. Henri-Poincaré 32, n 1, 91 (1980)

2. Bergère, M.C., Calan, C., de, Malbouisson, A.P.C.: Commun. Math. Phys. 62, 137 (1978)

3. Hepp, K.: Commun. Math. Phys. 2, 301 (1966)

4. Speer, E.: Generalized Feynman amplitudes. Princeton: Princeton University Press 1969

5. Bergère, M.C., Lam, Y.M.P.: J. Math. Phys. (NY) 17, 1546 (1976)

6. Bergère, M.C., David, F.: J. Math. Phys. (NY) 20, 1244 (1979)

Communicated by R. Stora

Received April 28, 1980; in revised form August 3, 1980 